\title{
DETERMINAÇÃO ESPECTROFOTOMÉTRICA SIMULTÂNEA DE COBRE E FERRO EM ÁLCOOL ETÍLICO COMBUSTÍVEL COM REAGENTES DERIVADOS DA FERROÍNA
}

Leonardo S. G. Teixeira*, João F. Brasileiro, Mário M. Borges Jr. e Paulo W. L. Cordeiro

Departamento de Engenharia e Arquitetura, Universidade Salvador, Av. Cardeal da Silva, 132, 40220-141 Salvador - BA, Brasil Sarah A. N. Rocha e Antonio C. Spínola Costa

Departamento de Química Analítica, Instituto de Química, Universidade Federal da Bahia, Campus Universitário de Ondina, 40170-290 Salvador-BA, Brasil

Recebido em 19/7/05; aceito em 28/9/05; publicado na web em 18/4/06

\begin{abstract}
SIMULTANEOUS SPECTROPHOTOMETRIC DETERMINATION OF IRON AND COPPER USING FERROIN REAGENTS. In the present work three ferroin reagents were studied for the simultaneous spectrophotometric determination of iron and copper: 1,10phenanthroline, 2,2'-bipyridine and 2,4,6-tri(2-pyridyl)-1,3,5-triazine. Effect of $\mathrm{pH}$, conditions, order reagent addition, interferences, amount of reagents, lineal range, sensitivity and stability of each system were compared. The 2,4,6-tri(2-pyridyl)-1,3,5-triazine can be used for determination of iron in the presence of copper with a detection limit of $5 \mu \mathrm{g} \mathrm{L}^{-1}$ and coefficient of variation of $2.0 \%$; However it was not possible to determine directly copper in the presence of iron with this reagent. 1,10-phenanthroline can be used for simultaneous determination of the metallic ions with detection limits of 7 and $8 \mu \mathrm{g} \mathrm{L}-1$ and coefficients of variation of 1.8 and $2.3 \%$ in the determination of iron and copper, respectively. The results showed also that 2,2'-bipyridine can be used for simultaneous determination of the metallic ions with detection limits of 11 and $32 \mu \mathrm{g} \mathrm{L}^{-1}$ and coefficients of variation of 1.9 and $2.5 \%$ in the determination of iron and copper, respectively. The reagents were used for spectrophotometric determination of iron and copper in ethanol fuel.
\end{abstract}

Keywords: alcohol fuel; ferroin; spectrophotometry.

\section{INTRODUÇÃO}

Os constantes aumentos dos preços do petróleo e as crescentes preocupações ambientais têm aumentado o interesse de muitos países na utilização de combustíveis alternativos de fontes renováveis. Entre os combustíveis alternativos aos derivados de petróleo, o álcool etílico combustível (AEC) tem se destacado no Brasil e vem sendo utilizado em misturas com a gasolina automotiva ou em automóveis movidos exclusivamente com esse combustível ${ }^{1}$. A partir do ano de 2004, pôde-se, inclusive, observar um aumento do consumo deste combustível devido ao surgimento dos automóveis bicombustíveis, que podem ser abastecidos tanto com gasolina como com etanol. Dessa forma, uma maior preocupação com a qualidade do AEC é pertinente, uma vez que combustíveis fora das especificações de qualidade trazem prejuízos diretos para o consumidor, através do mau funcionamento do motor automotivo, aumento do consumo de combustível e gastos com manutenção.

Entre os parâmetros para avaliação da qualidade do AEC está a contaminação com íons metálicos, que pode ocorrer no próprio processo de fabricação, bem como em tanques de transporte e armazenamento ${ }^{2,3}$. A Agência Nacional do Petróleo, Gás Natural e Biocombustíveis (ANP), através da Resolução no 36 de 17/12/2005, estabelece que o teor máximo de ferro no álcool etílico combustível hidratado não deve ser superior a $5 \mathrm{mg} \mathrm{kg}^{-1}$, enquanto que o teor de cobre no álcool etílico combustível anidro não deve ser superior a 0,07 $\mathrm{mg} \mathrm{kg}^{-1}{ }^{4}$. A mesma Portaria estabelece que as determinações dessas espécies no AEC devem ser realizadas usando-se a espectrometria de absorção atômica, através dos métodos NBR 11331 e NBR 10893. Entretanto, uma vez que nem sempre um espectrômetro de absorção atômica está disponível em laboratórios para análise de rotina, é interessante que métodos alternativos simples e de baixo custo sejam

*e-mail: leonardoteixeira@unifacs.br pesquisados e validados para determinação de cobre e ferro em AEC.

Entre as alternativas para determinação de íons metálicos de maneira simples e rápida destacam-se os métodos espectrofotométricos na região UV-Vis, devido à robustez e custo relativamente baixo ${ }^{5}$. Para determinação espectrofotométrica de cobre e ferro, podem-se destacar os reagentes da família da ferroína, devido aos complexos estáveis e seletivos que são formados com espécies iônicas desses metais ${ }^{6,7}$. Esses compostos contêm a estrutura $\alpha, \alpha^{\prime}$-di-imina que possui a seqüência de átomos ${ }^{8,9}-\mathrm{N}=\mathrm{C}-\mathrm{C}=\mathrm{N}-$. Outros íons metálicos também formam complexos com reagentes dessa classe, mas as características espectrais são diferentes o suficiente para que não interfiram em determinações espectrofotométricas de cobre e ferro ${ }^{10}$.

Reagentes da família da ferroína já foram usados para determinação de cobre e ferro nos mais variados tipos de amostras, incluindo amostras de água ${ }^{11,12}$, soluções alcalinas ${ }^{13}, \operatorname{solos}^{14} \mathrm{e}$, inclusive, matrizes alcoólicas ${ }^{15,16}$. Neste trabalho, três reagentes dessa família, conhecidos pela seletividade e sensibilidade para determinação espectrofotométrica de $\mathrm{Cu}$ e $\mathrm{Fe}$, foram avaliados para determinação simultânea destas espécies inorgânicas em AEC. Os reagentes testados foram 1,10-fenantrolina, 2,2'-bipiridina e a 2,4,6-tri(2-piridil)1,3,5-triazina para comparação de diferentes parâmetros analíticos, tais como condições de $\mathrm{pH}$, ordem de adição de reagentes, quantidade de reagente e tampão, faixa linear e estabilidade.

\section{PARTE EXPERIMENTAL}

\section{Reagentes}

Todos os reagentes usados foram de grau analítico e as soluções foram preparadas com água destilada-desionizada:

Soluções padrão de ferro e cobre $1000 \mathrm{mg} \mathrm{L}^{-1}$. Foram preparadas partindo de soluções contidas em ampolas Titrisol (Merck). 
As soluções de trabalho foram preparadas através de diluições apropriadas.

Solução tampão ácido acético/acetato de sódio 1,0 $\mathrm{mol} \mathrm{L}^{-1}(\mathrm{pH}$ 4,5). Foi preparada ajustando-se uma solução de acetato de sódio 1,0 mol L ${ }^{-1}$ com solução de ácido acético $1,0 \mathrm{~mol} \mathrm{~L}^{-1}$ para o $\mathrm{pH}$ desejado.

Solução tampão hidróxido de amônio/cloreto de amônio 1,0 mol $L^{-1}(p H 8,5)$. Foi preparada ajustando-se uma solução de hidróxido de amônio 1,0 mol L'-1 com solução de cloreto de amônio $1,0 \mathrm{~mol} \mathrm{~L}^{-1}$ para o $\mathrm{pH}$ desejado.

Solução tampão hexaminalácido clorídrico 1,0 $\mathrm{mol} \mathrm{L}^{-1}$ (pH 6,5). Foi preparada ajustando-se uma solução de hexamina $1,0 \mathrm{~mol} \mathrm{~L}^{-1} \mathrm{com}$ solução de ácido clorídrico $1,0 \mathrm{~mol} \mathrm{~L}^{-1}$ para o $\mathrm{pH}$ desejado.

Soluções de 1,10-fenantrolina, 2,2'-bipiridina e 2,4,6-tri(2piridil)-1,3,5-triazina 0,25\% $(\mathrm{m} / \mathrm{v})$. Dissolveu-se $0,25 \mathrm{~g}$ de cada um dos reagentes com etanol, sendo o volume de cada solução completado para $100 \mathrm{~mL}$ com etanol.

Solução de ácido ascórbico $1 \%(\mathrm{~m} / \mathrm{v})$. Dissolveu-se $1 \mathrm{~g}$ de ácido ascórbico em água e o volume foi completado para 100 mL com água.

\section{Instrumental}

As medidas de absorvância foram obtidas empregando-se espectrofotômetro UV-visível Varian modelo Cary 50, utilizando cela de $10 \mathrm{~mm}$ de caminho óptico. Os ajustes de $\mathrm{pH}$ foram feitos em potenciômetro Digimed modelo DM 20 (com eletrodo de vidro combinado DME-MV1).

\section{Preparação das amostras}

Amostras de álcool etílico hidratado combustível (AEHC) foram coletadas diretamente de bombas de abastecimento em postos revendedores de combustíveis de diferentes distribuidoras (Texaco, Shell, Esso, Satélite, CBPI, BR). Mediu-se $100 \mathrm{~mL}$ de cada amostra e aqueceu-se até secura. $\mathrm{O}$ resíduo obtido foi dissolvido com $1 \mathrm{~mL}$ de ácido clorídrico concentrado e diluído com água para $50 \mathrm{~mL}$ em balão volumétrico.

\section{Procedimento}

\section{Determinação espectrofotométrica de ferro e cobre com 1,10- fenantrolina}

Transferiu-se para um balão volumétrico de $25 \mathrm{~mL}$ uma alíquota de solução padrão ou de amostra contendo de 0,75 a 75,00 $\mu$ g de ferro ou cobre e acrescentou-se $1 \mathrm{~mL}$ da solução de ácido ascórbico. Adicionou-se, então, $4 \mathrm{~mL}$ da solução de 1,10-fenantrolina e $5 \mathrm{~mL}$ da solução tampão pH 4,5. Completou-se o volume com água, agitou-se e mediuse as absorvâncias dos complexos em 511 e 371 nm, utilizando um branco do reagente, preparado da mesma forma que as soluções de trabalho sem a adição dos analitos. Montou-se um sistema de equações com as curvas analíticas de calibração obtidas, para cálculo das concentrações dos respectivos íons metálicos nas amostras.

\section{Determinação espectrofotométrica de ferro e cobre com 2,2' - bipiridina}

Transferiu-se para um balão volumétrico de $25 \mathrm{~mL}$, uma alíquota de solução padrão ou de amostra contendo de 1,00 a 75,00 $\mu \mathrm{g}$ de ferro ou de 2,50 a 75,00 $\mu \mathrm{g}$ de cobre e acrescentou-se $1 \mathrm{~mL}$ da solução de ácido ascórbico. Adicionou-se, então, $4 \mathrm{~mL}$ da solução de 2,2‘bipiridina e $5 \mathrm{~mL}$ da solução tampão pH 6,5. Completou-se o volume com água, agitou-se e mediu-se as absorvâncias dos complexos em 523 e $435 \mathrm{~nm}$, utilizando um branco do reagente, preparado da mesma forma que as soluções de trabalho sem a adição dos analitos. Montou-se um sistema de equações com as curvas analíticas de calibração obtidas, para cálculo das concentrações dos respectivos íons metálicos.

\section{Determinação espectrofotométrica de ferro com 2,4,6-tri(2-piridil)- 1,3,5-triazina}

Transferiu-se para um balão volumétrico de $25 \mathrm{~mL}$ uma alíquota de solução padrão ou de amostra contendo de 1,00 a 75,00 $\mu \mathrm{g}$ de ferro e acrescentou-se $1 \mathrm{~mL}$ da solução de ácido ascórbico. Adicionou-se, então, 4 mL da solução de 2,4,6-tri(2-piridil)-1,3,5-triazina e $5 \mathrm{~mL}$ da solução tampão pH 8,5. Completou-se o volume com água, agitou-se e mediu-se a absorvância dos complexo em 595 nm, utilizando um branco do reagente, preparado da mesma forma que as soluções de trabalho sem a adição dos analitos. Calculou-se a concentração de ferro, utilizando a curva analítica de calibração.

\section{RESULTADOS E DISCUSSÃO}

\section{Determinação espectrofotométrica de ferro e cobre com 1,10- fenantrolina}

Em pH 4,5, a 1,10-fenantrolina apresentou absorção máxima em $365 \mathrm{~nm}$ e os complexos formados com $\mathrm{Fe}(\mathrm{II})$ e $\mathrm{Cu}$ (I) apresentaram absorção máxima em 511 e 371 nm, respectivamente. O ligante não apresentou absorção em 511 e $371 \mathrm{~nm}$ em soluções tamponadas em pH 4,5. A determinação de ferro em $511 \mathrm{~nm}$ é isenta da interferência de cobre. Entretanto, para a determinação de cobre foi empregada a propriedade aditiva das absorvâncias, visto que o complexo formado com ferro absorve também em $371 \mathrm{~nm}$.

Para estudo da estabilidade do sistema, as leituras das absorvâncias foram realizadas logo após a mistura dos reagentes e aferição dos volumes nos balões volumétricos. Verificou-se que tanto o reagente como os dois complexos metálicos foram estáveis por pelo menos $24 \mathrm{~h} \mathrm{em} \mathrm{pH} \mathrm{4,5.}$

Realizando o estudo do efeito da acidez no sistema, verificou-se que os complexos da 1,10-fenantrolina com ferro e cobre apresentam absorções máximas nas faixas de $\mathrm{pH}$ compreendidas entre 3,0 a 10,0 e de 4,0 a 7,0, respectivamente. Escolheu-se, então, tampão ácido acético/ acetato de sódio ajustado em $\mathrm{pH}$ 4,5 para controle do $\mathrm{pH}$ das soluções.

O efeito da concentração do tampão acetato de sódio/ácido acético (pH 4,5) sobre os complexos da 1,10-fenantrolina com o Fe(II) e Cu(I) foi estudado e os resultados demonstraram que a quantidade de acetato não modificou o sinal da absorvância de maneira significativa dentro da faixa de $0,040 \mathrm{a} 0,40 \mathrm{~mol} \mathrm{~L}^{-1}$. Quanto à presença da 1,10-fenantrolina no sistema, verificou-se que concentrações de reagente superiores a $1,0 \times 10^{-3} \mathrm{~mol} \mathrm{~L}^{-1}$ eram suficientes para garantir completa complexação dos íons metálicos e, conseqüentemente, sinais de absorvância máximos e estáveis nas faixas de concentrações trabalhadas.

Uma vez que os complexos formados com a 1,10-fenantrolina são com $\mathrm{Fe}(\mathrm{II})$ e $\mathrm{Cu}(\mathrm{I})$, utilizou-se ácido ascórbico como agente redutor. Dessa forma, garantiu-se que todo ferro e cobre estivessem no sistema nas formas reduzidas. O efeito da concentração do ácido ascórbico no sistema foi estudado e os resultados mostraram que, dentro da faixa de 1,1 a $4,5 \times 10^{-3} \mathrm{~mol} \mathrm{~L}^{-1}$, a quantidade de ácido ascórbico não influencia de maneira significativa os sinais de absorvância.

A influência da ordem de adição de reagentes sobre a formação dos complexos do $\mathrm{Fe}(\mathrm{II})$ e $\mathrm{Cu}(\mathrm{I})$ foi estudada e verificou-se que melhores sinais de absorvância são obtidos quando os reagentes são adicionados na seguinte ordem: solução padrão ou amostra, redutor, reagente cromogênico e solução tampão.

As curvas analíticas foram preparadas segundo o procedimento experimental e verificou-se obediência à lei de Beer na faixa de concentração de 0,030 até $3,0 \mu \mathrm{g} \mathrm{mL}^{-1}$ para ferro e cobre. As equações obtidas foram: $A_{\mathrm{Fe}(511)}=0,207[\mathrm{Fe}](\mathrm{R}=0,9992), \mathrm{A}_{\mathrm{Fe}(371)}=0,019$ 
$[\mathrm{Fe}]+0,001(\mathrm{R}=0,9987)$ e $\mathrm{A}_{\mathrm{Cu}(371)}=0,190[\mathrm{Cu}](\mathrm{R}=0,9990)$. A concentração de ferro foi obtida diretamente através da absorvância medida em $511 \mathrm{~nm}$. Para o cobre, a concentração foi encontrada baseando-se na propriedade aditiva da absorvância medida em $371 \mathrm{~nm}$ :

$\mathrm{C}_{\mathrm{Fe}}=\frac{\mathrm{A}_{511}}{0,207}$

$\mathrm{C}_{\mathrm{Cu}}=\frac{\mathrm{A}_{366}-\left(0,019 \mathrm{C}_{\mathrm{Fe}}+0,001\right)}{0,190}$

Os limites de detecção $(3 \sigma)$ e os coeficientes de variação para determinação de ferro e cobre foram estimados em 7 e $8 \mu \mathrm{g} \mathrm{L}^{-1}$ e 1,8 e $2,3 \%$, respectivamente.

\section{Determinação espectrofotométrica de ferro e cobre com 2,2'- bipiridina}

Em pH 6,5, os complexos da 2,2‘-bipiridina formados com Fe(II) e $\mathrm{Cu}(\mathrm{I})$ apresentaram absorções máximas em 523 e 435 nm, respectivamente. Neste mesmo $\mathrm{pH}$, o ligante apresentou absorção máxima em 280 nm e não apresentou absorção em 523 e 435 nm. Para determinação dos dois íons metálicos foi empregada a propriedade aditiva das absorvâncias, visto que os complexos absorvem nos dois comprimentos de onda.

Para estudo da estabilidade do sistema, as leituras das absorvâncias foram realizadas logo após a mistura dos reagentes e aferição dos volumes nos balões volumétricos. Verificou-se que tanto o reagente como os dois complexos metálicos foram estáveis por pelo menos $24 \mathrm{~h}$ em pH 6,5.

Realizando o estudo do efeito da acidez no sistema, verificou-se que os complexos da 2,2'-bipiridina com ferro e cobre apresentam melhores sinais de absorvância nas faixas de $\mathrm{pH}$ compreendidas entre 3,0 a 8,0 e de 4,5 a 7,0, respectivamente. Escolheu-se, então, solução tampão hexamina/ácido clorídrico ajustado em $\mathrm{pH}$ 6,5 para controle do $\mathrm{pH}$ das soluções.

O efeito da concentração da solução tampão $(\mathrm{pH}$ 6,5) sobre os complexos da 2,2'-bipiridina com o $\mathrm{Fe}(\mathrm{II})$ e $\mathrm{Cu}(\mathrm{I})$ foi estudado e os resultados demonstraram que a quantidade de hexamina não modificou o sinal da absorvância de maneira significativa dentro da faixa de 0,040 a $0,40 \mathrm{~mol} \mathrm{~L}^{-1}$. Quanto à presença da 2,2'bipiridina no sistema, verificou-se que concentrações de reagente superiores a $1,0 \times 10^{-3} \mathrm{~mol} \mathrm{~L}^{-1}$ eram suficientes para garantir complexação completa dos respectivos íons metálicos com sinais de absorvância máximos e estáveis nas faixas de concentrações trabalhadas.

Os complexos formados com a 2,2'-bipiridina são com as espécies $\mathrm{Fe}(\mathrm{I})$ e $\mathrm{Cu}$ (II). Dessa forma, utilizou-se ácido ascórbico como agente redutor para garantir que o ferro e cobre estivessem no sistema nessas formas. O efeito da concentração do ácido ascórbico no sistema foi estudado e os resultados mostraram que dentro da faixa de 1,1 a $4,5 \times 10^{-3} \mathrm{~mol} \mathrm{~L}^{-1}$, a quantidade de ácido ascórbico não influencia de maneira significativa os sinais de absorvância.

A influência da ordem de adição de reagentes sobre a formação dos complexos foi estudada e verificou-se que melhores sinais de absorvância são obtidos quando os reagentes são adicionados na seguinte ordem: solução padrão ou amostra, redutor, reagente cromogênico e solução tampão.

As curvas analíticas foram preparadas segundo o procedimento experimental e verificou-se obediência à lei de Beer na faixa de concentração de 0,040 até $3,0 \mu \mathrm{g} \mathrm{mL}^{-1}$ e 0,10 até $3,0 \mu \mathrm{g} \mathrm{mL}^{-1}$ para o ferro e cobre, respectivamente. As equações obtidas foram: $\mathrm{A}_{\mathrm{Fe}(522)}=$
$0,162[\mathrm{Fe}](\mathrm{R}=0,9999), \mathrm{A}_{\mathrm{Fe}(435)}=0,040[\mathrm{Fe}]+0,003(\mathrm{R}=0,9991)$, $\mathrm{A}_{\mathrm{Cu}(522)}=0,018[\mathrm{Cu}]-0,001(\mathrm{R}=0,9987)$ e $\mathrm{A}_{\mathrm{Cu}(435)}=0,050[\mathrm{Cu}]-$ $0,002(R=0,9989)$. As concentrações de ferro e cobre nas amostras foram encontradas utilizando a propriedade aditiva da absorvância:

$\begin{aligned} C_{\mathrm{Fe}} & =\frac{\mathrm{A}_{522}-\left(0,018 \mathrm{C}_{\mathrm{Cu}}-0,001\right)}{0,162} \\ \mathrm{C}_{\mathrm{Cu}} & =\frac{\mathrm{A}_{430}-\left(0,040 \mathrm{C}_{\mathrm{Fe}}+0,001\right)}{0,050}\end{aligned}$

Os limites de detecção $(3 \sigma)$ e os coeficientes de variação, para determinação de ferro e cobre, foram estimados em 11 e $32 \mu \mathrm{g} \mathrm{L}^{-1} \mathrm{e}$ 1,9 e $2,5 \%$, respectivamente.

\section{Determinação espectrofotométrica de ferro com 2,4,6-tri(2- piridil)-1,3,5-triazina}

Os complexos do $\mathrm{Fe}(\mathrm{II})$ e $\mathrm{Cu}$ (I) com a 2,4,6-tri(2-piridil)-1,3,5triazina apresentaram absorções máximas em 595 e 340 nm, respectivamente, em meio tamponado em $\mathrm{pH} 8,5$. A determinação de ferro em $595 \mathrm{~nm}$ é realizada empregando a propriedade aditiva das absorvâncias, visto que o complexo da 2,4,6-tri(2-piridil)-1,3,5-triazina com o cobre também absorve neste comprimento de onda. No comprimento de onda de absorção máxima do complexo do cobre (340 $\mathrm{nm}$ ), o complexo do ferro e o próprio ligante absorvem intensamente. A 2,4,6-tri(2-piridil)-1,3,5-triazina apresentou absortividade molar de $3,2 \times 10^{2} \mathrm{~L} \mathrm{~mol}^{-1} \mathrm{~cm}^{-1}$, o complexo do ligante com o ferro apresentou absortividade molar de $1,45 \times 10^{4} \mathrm{~L} \mathrm{~mol}^{-1} \mathrm{~cm}^{-1}$ e o complexo com o cobre apresentou absortividade molar igual a $4,96 \times 10^{3} \mathrm{~L} \mathrm{~mol}^{-1} \mathrm{~cm}^{-1}$ em $340 \mathrm{~nm}$. Como se pode observar, além do próprio ligante absorver em $340 \mathrm{~nm}$, a absortividade do complexo com o ferro é maior que a do cobre. Este fato dificultou a determinação simultânea de cobre e ferro com este reagente. De qualquer forma, deu-se continuidade ao estudo para determinação das condições reacionais ótimas para determinação de cobre.

Para estudo da estabilidade do sistema, as leituras das absorvâncias foram realizadas logo após a mistura dos reagentes e aferição dos volumes nos balões volumétricos. Verificou-se que tanto o reagente como os dois complexos metálicos mostraram ser estáveis por pelo menos $24 \mathrm{~h}$ em $\mathrm{pH} 8,0$.

Realizando o estudo do efeito da acidez no sistema, verificou-se que os complexos da 2,4,6-tri(2-piridil)-1,3,5-triazina com ferro e cobre apresentam absorção máxima em $\mathrm{pH}$ na faixa compreendida entre 3,0 a 8,5 e de 7,0 a 8,5, respectivamente. Escolheu-se, então, solução tampão hidróxido de amônio/cloreto de amônio ajustado em pH 8,0 para controle do $\mathrm{pH}$ das soluções.

O efeito da concentração da solução tampão $(\mathrm{pH} \mathrm{8,0)}$ sobre os complexos da 2,4,6-tri(2-piridil)-1,3,5-triazina com o $\mathrm{Fe}(\mathrm{II})$ e $\mathrm{Cu}(\mathrm{I})$ foi estudado e os resultados demonstraram que a quantidade de amônio não influenciou o sinal da absorvância de maneira significativa dentro da faixa de $0,040 \mathrm{a} 0,40 \mathrm{~mol} \mathrm{~L}^{-1}$. Quanto à presença da 2,4,6-tri(2-piridil)-1,3,5-triazina no sistema, verificou-se que concentrações de reagente superiores a $1,0 \times 10^{-3} \mathrm{~mol} \mathrm{~L}^{-1}$ eram suficientes para garantir complexação completa dos íons metálicos e, conseqüentemente, sinais de absorvância máximos e estáveis nas faixas de concentrações trabalhadas.

Utilizou-se ácido ascórbico como agente redutor para garantir que o ferro e cobre estivessem no sistema na forma de $\mathrm{Fe}(\mathrm{II})$ e $\mathrm{Cu}$ (I), uma vez que os complexos formados com a 2,4,6-tri(2-piridil)-1,3,5-triazina são com as formas reduzidas de ferro e cobre. O efeito da concentração do ácido ascórbico no sistema foi estudado e os resultados mos- 
traram que dentro da faixa de 1,1 a $4,5 \times 10^{-3} \mathrm{~mol} \mathrm{~L}^{-1}$, a quantidade de ácido ascórbico não influencia de maneira significativa os sinais de absorvância.

Assim como observado com a 2,2'-bipiridina e a 1,10fenantrolina, quando se realizou o estudo da influência da ordem de adição de reagentes sobre a formação dos complexos, verificouse que melhores sinais de absorvância são obtidos quando os reagentes são adicionados na seguinte ordem: solução padrão ou amostra, redutor, reagente cromogênico e solução tampão.

As curvas analíticas foram preparadas segundo o procedimento experimental, verificando-se obediência à lei de Beer na faixa de concentração de 0,040 até $3,0 \mu \mathrm{g} \mathrm{mL}^{-1}$ e 0,30 até $3,0 \mu \mathrm{g} \mathrm{mL} \mathrm{m}^{-1}$ para o ferro e cobre, respectivamente. As equações obtidas foram: $\mathrm{A}_{\mathrm{Fe}(595)}=$ $0,396[\mathrm{Fe}](\mathrm{R}=0,9999), \mathrm{A}_{\mathrm{Fe}(340)}=0,259[\mathrm{Fe}](\mathrm{R}=0,9992), \mathrm{A}_{\mathrm{Cu}(595)}=$ $0,024[\mathrm{Cu}]-0,002(\mathrm{R}=0,9989)$ e $\mathrm{A}_{\mathrm{Cu}(340)}=0,078[\mathrm{Cu}]+0,100(\mathrm{R}=$ 0,9987). As concentrações de ferro nas amostras foram encontradas utilizando a propriedade aditiva da absorvância:

$\mathrm{C}_{\mathrm{Fe}}=\frac{\mathrm{A}_{595}-\left(0,024 \mathrm{C}_{\mathrm{Cu}}-0,002\right)}{0,396}$

Tentativas para determinação de cobre em amostras contendo ferro foram feitas utilizando a Equação:

$\mathrm{C}_{\mathrm{Cu}}=\frac{\mathrm{A}_{340}-\left(0,254 \mathrm{C}_{\mathrm{Fe}}+0,100\right)}{0,078}$

Os limites de detecção $(3 \sigma)$ e os coeficientes de variação, para determinação de ferro e cobre, foram estimados em 5 e $77 \mu \mathrm{g} \mathrm{L}^{-1} \mathrm{e}$ 2,0 e $2,8 \%$, respectivamente.

\section{Aplicação}

Para avaliação da seletividade, visando a aplicação dos reagentes na determinação do ferro e cobre em amostras de AEC, foram preparadas soluções contendo essas duas espécies juntamente com os possíveis interferentes. Nesse sentido, foram preparadas soluções contendo $\mathrm{Na}, \mathrm{K}, \mathrm{Ca}, \mathrm{Mg}, \mathrm{Ba}$ em concentrações 100 vezes superiores às concentrações de ferro e cobre e $\mathrm{Mn}, \mathrm{Co}, \mathrm{Ag}, \mathrm{Ni}, \mathrm{Zn}, \mathrm{Cd}, \mathrm{Al}$ em concentrações 10 vezes superiores às concentrações de ferro e cobre. Testou-se também a influência da presença de cloreto, brometo, iodeto, sulfato, nitrato, carbonato e acetato em concentrações 1000 vezes maiores que as concentrações de cobre e ferro. Fixou-se o limite de tolerância de erro em $\pm 5 \%$ do sinal de absorvância obtido para soluções contendo $2,0 \mu \mathrm{g} \mathrm{mL}^{-1}$ de ferro e cobre. Verificou-se que para as concentrações estudadas, não ocorreu interferência de nenhuma das espécies testadas.

Na Tabela 1 estão sumarizados alguns parâmetros analíticos para determinações espectrofotométricas com os três reagentes testados. Os métodos desenvolvidos para cada um dos três reagentes foram testados preliminarmente em amostras sintéticas contendo diferentes concentrações de cobre e ferro, para verificação da viabilidade de aplicação dos reagentes para determinação simultânea dos respectivos íons metálicos, usando-se as equações propostas. Os resultados obtidos como média de três determinações com os respectivos desvios a 95\% de nível de confiança estão apresentados na Tabela 2. Conforme pode ser observado, os resultados encontrados para determinação simultânea de ferro e cobre com 1,10-fenantrolina e 2,2'bipiridina foram satisfatórios. Obtiveram-se também bons resultados para a determinação de ferro com a 2,4,6-tri(2-piridil)-1,3,5triazina, entretanto, a determinação de cobre na presença de ferro com este reagente mostrou sofrer grande interferência. Dessa forma, recomenda-se que a 2,4,6-tri(2-piridil)-1,3,5-triazina seja utili-

Tabela 1. Características analíticas dos métodos espectrofotométricos propostos para determinação de cobre e ferro

\begin{tabular}{|c|c|c|c|c|c|c|c|c|c|}
\hline \multirow[t]{2}{*}{ Reagente } & \multirow{2}{*}{ Fórmula Estrutural } & \multicolumn{4}{|c|}{ Determinação de Ferro } & \multicolumn{4}{|c|}{ Determinação de Cobre } \\
\hline & & $\lambda_{\max ,} n \mathrm{~nm}$ & $\begin{array}{c}\varepsilon \\
\mathrm{L} \mathrm{mol}^{-1} \mathrm{~cm}^{-1}\end{array}$ & $\mathrm{pH}$ & $\begin{array}{c}\mathrm{LD} \\
\mu \mathrm{g} \mathrm{L}^{-1}(3 \sigma)\end{array}$ & $\lambda_{\max ,} n$ & $\begin{array}{c}\varepsilon \\
\mathrm{L} \mathrm{mol}^{-1} \mathrm{~cm}^{-1}\end{array}$ & $\mathrm{pH}$ & $\begin{array}{l}\mathrm{LD} \\
\mathrm{L}^{-1}(3 \sigma)\end{array}$ \\
\hline 1,10-fenantrolina & & 511 & $1,16 \times 10^{4}$ & $3,0-10,0$ & 7 & 371 & $1,21 \times 10^{4}$ & $4,0-7,0$ & 8 \\
\hline 2,2'-bipiridina & & 523 & $9,06 \times 10^{4}$ & $3,0-8,0$ & 11 & 435 & $3,16 \times 10^{4}$ & $4,5-7,0$ & 32 \\
\hline $\begin{array}{l}\text { 2,4,6-tri(2-piridil)- } \\
\text { 1,3,5-triazina }\end{array}$ & & 595 & $2,20 \times 10^{4}$ & $3,0-8,5$ & 5 & 340 & $4,96 \times 10^{3}$ & $7,0-8,5$ & 77 \\
\hline
\end{tabular}

Tabela 2. Determinação de ferro e cobre $\left(\mathrm{mg} \mathrm{L}^{-1}\right)$ em amostras sintéticas utilizando reagentes da família da ferroína (n=3)

\begin{tabular}{|c|c|c|c|c|c|c|c|c|}
\hline \multirow[t]{2}{*}{ Amostra } & \multicolumn{2}{|c|}{$\begin{array}{l}\text { Quantidade Adicionada } \\
\qquad\left(\mathrm{mg} \mathrm{L}^{-1}\right)\end{array}$} & \multicolumn{2}{|c|}{$\begin{array}{l}\text { Quantidade Encontrada } \\
\text { 1,10-fenantrolina }\end{array}$} & \multicolumn{2}{|c|}{$\begin{array}{c}\text { Quantidade Encontrada } \\
\text { 2,2'-bipiridina }\end{array}$} & \multicolumn{2}{|c|}{$\begin{array}{c}\text { Quantidade Encontrada } \\
\text { 2,4,6-tri(2-piridil)-1,3,5-triazina }\end{array}$} \\
\hline & Ferro & Cobre & Ferro & Cobre & Ferro & Cobre & Ferro & Cobre \\
\hline 1 & 0,10 & 0,10 & $0,10 \pm 0,01$ & $0,11 \pm 0,01$ & $0,09 \pm 0,1$ & $0,08 \pm 0,02$ & $0,09 \pm 0,02$ & $0,18 \pm 0,6$ \\
\hline 2 & 0,50 & 0,50 & $0,48 \pm 0,02$ & $0,51 \pm 0,03$ & $0,51 \pm 0,02$ & $0,49 \pm 0,01$ & $0,49 \pm 0,01$ & $0,75 \pm 0,8$ \\
\hline 3 & 0,20 & 1,0 & $0,19 \pm 0,03$ & $1,1 \pm 0,2$ & $0,22 \pm 0,02$ & $1,0 \pm 0,3$ & $0,22 \pm 0,03$ & $2,2 \pm 0,4$ \\
\hline 4 & 2,0 & 0,5 & $2,0 \pm 0,1$ & $0,49 \pm 0,03$ & $2,2 \pm 0,4$ & $0,53 \pm 0,04$ & $2,1 \pm 0,3$ & $0,82 \pm 0,05$ \\
\hline 5 & 0,10 & 0,50 & $0,11 \pm 0,02$ & $0,51 \pm 0,02$ & $0,11 \pm 0,02$ & $0,52 \pm 0,02$ & $0,10 \pm 0,01$ & $0,79 \pm 0,09$ \\
\hline
\end{tabular}


Tabela 3. Características físico-químicas das amostras coletadas de álcool etílico combustível

\begin{tabular}{|c|c|c|c|c|c|c|c|}
\hline Amostra & Aparência & Cor & $\mathrm{pH}$ & $\begin{array}{c}\text { Condutividade } \\
\text { Elétrica, } \\
\mu \mathrm{S} / \mathrm{m}\end{array}$ & $\begin{array}{c}\text { Massa } \\
\text { Específica, } \\
\mathrm{kg} / \mathrm{m}^{3}\end{array}$ & $\begin{array}{c}\text { Teor } \\
\text { Alcoólico, } \\
\text { oINPM }\end{array}$ & $\begin{array}{c}\text { Teor de } \\
\text { Hidrocarbonetos, } \\
\text { \% vol. }\end{array}$ \\
\hline $\mathrm{A}$ & Límpido e isento de impurezas & Incolor & 8,0 & 263 & 809,7 & 93,1 & $<1$ \\
\hline $\mathrm{B}$ & Límpido e isento de impurezas & Incolor & 7,6 & 459 & 811,0 & 92,6 & $<1$ \\
\hline $\mathrm{C}$ & Límpido e isento de impurezas & Incolor & 7,1 & 320 & 811,0 & 92,6 & $<1$ \\
\hline $\mathrm{D}$ & Límpido e isento de impurezas & Incolor & 6,8 & 339 & 809,6 & 93,1 & $<1$ \\
\hline $\mathrm{E}$ & Límpido e isento de impurezas & Incolor & 6,3 & 212 & 811,0 & 92,6 & $<1$ \\
\hline $\mathrm{F}$ & Límpido e isento de impurezas & Incolor & 6,9 & 245 & 808,9 & 93,3 & $<1$ \\
\hline $\begin{array}{l}\text { Especificação } \\
\text { da ANP para } \\
\text { o AEHC }\end{array}$ & Límpido e isento de impurezas & Incolor & 6,0 a 8,0 & $500, \max$ & 805,0 a 811,0 & 92,6 a 94,7 & 3, max. \\
\hline
\end{tabular}

Tabela 4. Determinação de ferro e cobre $\left(\mathrm{mg} \mathrm{L}^{-1}\right)$ em amostras de álcool etílico hidratado combustível (n=3)

\begin{tabular}{|c|c|c|c|c|c|c|c|c|}
\hline \multirow[t]{2}{*}{ Amostra } & \multicolumn{2}{|c|}{$\begin{array}{c}\text { Quantidade Encontrada } \\
\text { FAAS }\end{array}$} & \multicolumn{2}{|c|}{$\begin{array}{l}\text { Quantidade Encontrada } \\
1,10 \text {-fenantrolina }\end{array}$} & \multicolumn{2}{|c|}{$\begin{array}{l}\text { Quantidade Encontrada } \\
2,2^{‘} \text {-bipiridina }\end{array}$} & \multicolumn{2}{|c|}{$\begin{array}{l}\text { Quantidade Encontrada } \\
\text { 2,4,6-tri(2-piridil)-1,3,5-triazina }\end{array}$} \\
\hline & Ferro & Cobre & Ferro & Cobre & Ferro & Cobre & Ferro & Cobre \\
\hline $\mathrm{A}$ & $0,10 \pm 0,03$ & $0,09 \pm 0,01$ & $0,06 \pm 0,02$ & $0,06=$ & $0,07 \pm 0,02$ & $0,11=$ & 0,02 & $11 \mathrm{a}$ \\
\hline $\mathrm{B}$ & $0,25 \pm 0,05$ & $0,10 \pm 0,02$ & $0,19 \pm 0,02$ & $0,14 \pm 0,03$ & $0,18 \pm 0,01$ & $0,12 \pm 0,02$ & $0,19 \pm 0,03$ & na \\
\hline $\mathrm{C}$ & $0,17 \pm 0,03$ & $0,10 \pm 0,03$ & $0,14 \pm 0,02$ & $0,11 \pm 0,01$ & $0,15 \pm 0,03$ & $0,11 \pm 0,03$ & $0,13 \pm 0,01$ & na \\
\hline $\mathrm{D}$ & $0,33 \pm 0,04$ & $0,17 \pm 0,03$ & $0,26 \pm 0,04$ & $0,16 \pm 0,03$ & $0,24 \pm 0,03$ & $0,19 \pm 0,03$ & $0,28 \pm 0,03$ & na \\
\hline $\mathrm{E}$ & $0,32 \pm 0,05$ & $0,16 \pm 0,03$ & $0,26 \pm 0,02$ & $0,17 \pm 0,02$ & $0,28 \pm 0,04$ & $0,17 \pm 0,03$ & $0,33 \pm 0,04$ & na \\
\hline $\mathrm{F}$ & $0,15 \pm 0,02$ & $0,06 \pm 0,02$ & $0,16 \pm 0,03$ & $0,09 \pm 0,02$ & $0,12 \pm 0,02$ & $0,07 \pm 0,01$ & $0,14 \pm 0,03$ & na \\
\hline
\end{tabular}

na - não analisado

zada apenas para a determinação de ferro, quando o cobre estiver presente na amostra.

Os métodos estudados foram aplicados para a determinação de ferro e cobre em 6 amostras de álcool etílico combustível coletadas em diferentes postos de combustíveis. Algumas características físico-químicas das amostras foram determinadas, conforme a Portaria da ANP $\mathrm{N}^{\circ} 45$ de 16 de março de 2001, e são apresentadas na Tabela 3. Os resultados obtidos para determinação de ferro e cobre com cada um dos três reagentes espectrofotométricos e os resultados obtidos usando a espectrometria de absorção atômica com chama (FAAS) podem ser observados na Tabela 4. Os resultados estão expressos como média de 3 determinações independentes para cada uma das seis amostras, com os respectivos desvios a $95 \%$ de nível de confiança.

\section{CONCLUSÕES}

Os complexos de ferro e cobre formados com a 1,10-fenantrolina, 2,2'-bipiridina e com a 2,4,6-tri(2-piridil)-1,3,5-triazina apresentaramse estáveis em uma boa faixa de $\mathrm{pH}$ e os respectivos métodos espectrofotométricos apresentaram uma boa linearidade, reprodutibilidade e sensibilidade. Os métodos desenvolvidos com os três reagentes apresentaram bons limites de detecção para a determinação de ferro. Para a determinação de cobre, a 1,10-fenantrolina foi o reagente que apresentou melhor sensibilidade, enquanto que a determinação desse metal com a 2,4,6-tri(2-piridil)-1,3,5-triazina mostrou sofrer grande interferência na presença de ferro. Com com a 1,10fenantrolina e a 2,2 ${ }^{6}$-bipiridina foi possível a realização de determinações espectrofotométricas simultâneas de $\mathrm{Cu}$ e Fe em amostras de álcool combustível de maneira seletiva, sensível e simples.

\section{AGRADECIMENTOS}

À CAPES, ANP/PRH23, CNPq e FINEP/CTPetro pelo apoio financeiro.

\section{REFERÊNCIAS}

1. Teixeira, L. S. G.; Leão, E. S.; Dantas, A. F.; Pinheiro, H. L. C.; Costa, A. C. S.; de Andrade, J. B.; Talanta 2004, 64, 711.

2. Saint'Pierre, T.; Aucelio, R. Q.; Curtis, A. J.; Microchem. J. 2003, 75, 59.

3. Lechner-Knoblauch, U.; Heitz, E.; Electrochim. Acta 1987, 32, 901.

4. BRASIL. Portaria ANP n 2 de 16 de janeiro de 2002. Estabelece as especificações para comercialização do Álcool Etílico Anidro Combustível (AEAC) e do Álcool Etílico Hidratado Combustível (AEHC) em todo o território nacional e define obrigações dos agentes econômicos sobre o controle de qualidade do produto.

5. Rocha, F. R. P.; Teixeira, L. S. G.; Quim. Nova 2004, 27, 807.

6. Schilt, A. A.; Mohamed, N.; Talanta 1979, 26, 85.

7. Schilt, A. A.; Smith, G. F.; Anal. Chem. Acta 1957, 16, 401.

8. Stephen, W. I.; Talanta 1969, 16, 939.

9. Wilkins, D. H.; Smith, F.; Anal. Chem. Acta 1953, 9, 538.

10. Ueno, K.; Iamamura, T.; Cheng, K. L.; Handbook of Organic Analytical Reagents, CRC Press: Florida, 1982, p. 371.

11. Kawakubo, S.; Naito, A.; Fujihara, A.; Iwatsuki, M.; Anal. Sci. 2004, 20, 1159.

12. Toral, M. I,; Richter, P.; Rodríguez, C.; Talanta 1997, 45, 147.

13. Dunbar, W. E.; Schilt, A. A.; Talanta 1972, 19, 1025.

14. Gomes, D. M. C.; Segundo, M. A.; Lima, J. L. F. C.; Rangel, A. O. S. S.; Talanta 2005, 66, 703.

15. Gerber, L.; Claassen, R. I.; Boruff, C. S.; Ind. Eng. Chem. 1942, 14, 364.

16. Ampan, P.; Lapanantnoppakhun, S.; Sooksamiti, P.; Jakmunee, J.; KradtapHartwell, S.; Jayasvati, S.; Christian, G. D.; Grudpan, K.; Talanta 2002, $58,1327$. 